\title{
Imaging of Sciatic Lymphoma
}

Alberto Priori MD, PhD; Serena Magno, MD; Laura Campiglio, MD; Elena Lovati, MD; Luca

Tagliabue, MD.

Alberto Priori, Dipartimento di Scienze della Salute, Università degli Studi di Milano, ASST Santi Paolo e Carlo, Fondazione IRCCS Cà Granda, Clinica Neurologica III, Milan, Italy

Serena Magno, University of Milan, Italy Laura Campiglio, ASST Santi Paolo e Carlo, Neurology Unit, Milan, Italy Elena Lovati, ASST Santi Paolo e Carlo, Radiology Unit, Milan, Italy

Luca Tagliabue, ASST Santi Paolo e Carlo, Nuclear Medicine Unit, Milan, Italy

Word count paper: 274

Number of references: 3

Number of figures: 2

Key words: neuropathy; sciatic nerve; lymphoma; MRI; DWI.

Corresponding Author: Prof. Alberto Priori, Clinica Neurologica III, ASST Santi Paolo e Carlo, Via Antonio di Rudinì 8, Milano, Italy, 20142, alberto.priori@unimi.it

Financial Disclosure and Funding: none of the authors has any conflict of interest to disclose. All authors have read the Journal's position on issues involved in ethical publication and affirm that this report is consistent with those guidelines.

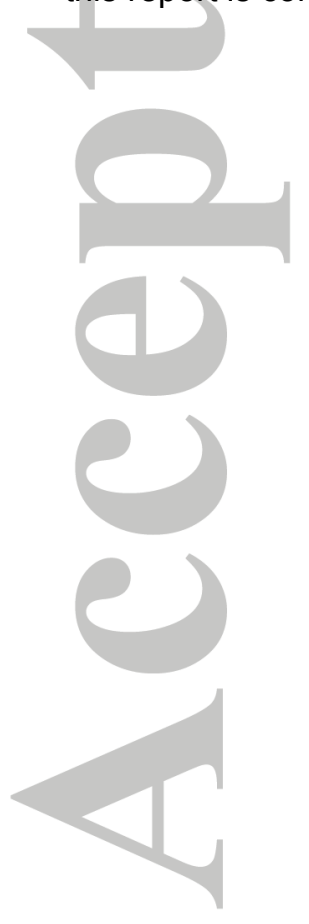

This article has been accepted for publication and undergone full peer review but has not been through the copyediting, typesetting, pagination and proofreading process which may lead to differences between this version and the Version of Record. Please cite this article as an 'Accepted Article', doi: 10.1002/mus.25698 


\section{Imaging of Sciatic Lymphoma}

Peripheral nerve infiltration in lymphoma is a rare condition. The most common clinical manifestation is painful polyradiculopathy, ${ }^{1}$ but presentation can vary, mimicking other common causes of neuropathy. Therefore, diagnosis is often challenging. Here we provide imaging of a 72year-old woman who presented with right foot drop due to a primary sciatic nerve lymphoma. Neurological examination revealed severe weakness in the distribution of the right common peroneal and tibial nerves, sensory impairment in the sural and deep peroneal nerve distributions, an absent right ankle jerk, and a palpable mass in the posterior lower right thigh. Magnetic resonance imaging (Figure 1) showed a fusiform bilobed mass with a medial necrotic and a lateral solid component, predominantly hypo-isointense to muscle $(3.8 \times 3 \times 10.7 \mathrm{~cm})$ in T1-weighted sequences without and with gadolinium enhancement, and heterogeneous signal in T2-weighted sequences. Diffusion-weighted imaging (DWI) and apparent diffusion coefficient (ADC) sequences showed restricted diffusion with low ADC values $\left(0.8 \mathrm{~mm}^{2} / \mathrm{s}\right)$. Because the diffusivity of water molecules is restricted by high cellularity, these findings suggested increased cellularity ${ }^{2,3}$ and malignancy ${ }^{2}$.

A computed tomography scan (Figures 2A, 2B), ${ }^{18}$ fluorodeoxyglucose positron emission tomography $\left({ }^{18} \mathrm{FDG}\right)-\mathrm{PET}$ (Figures $\left.2 \mathrm{C}, 2 \mathrm{D}\right)$, and fusion-PET/CT $\left({ }^{18} \mathrm{FDG}\right)-\mathrm{PET} / \mathrm{CT}$ (Figures 2E, 2F) showed increased uptake and enlargement of the right sciatic nerve, including the tibial and peroneal branches. An open surgical nerve biopsy confirmed a diffuse large B-cell sciatic lymphoma. The patient was treated with systemic chemotherapy. After 3 months, no disease progression was evident, but the neurological deficit remained unchanged. Although nerve biopsy represents the gold standard for diagnosis, MRI with functional sequences (DWI and $A D C$ ) and PET can play a pivotal role in the early recognition of peripheral nerve lymphoma.

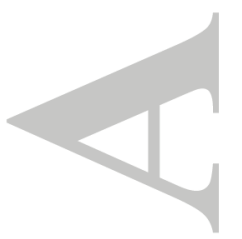




\section{References}

1. Lahoria R, Dyck PJ, Macon WR, Crum BA, Spinner RJ, Amrami KK, et al.

Neurolymphomatosis: A report of 2 cases representing opposite ends of the clinical spectrum. Muscle Nerve 2015;52(3):449-54. doi:10.1002/mus.24646. Epub 2015 May 14.

2. Schob S, Meyer J, Gawlitza M, Frydrychowicz C, Muller W, Preuss M, et al. DiffusionWeighted MRI Reflects Proliferative Activity in Primary CNS Lymphoma. PLoS One. 2016 Aug 29;11(8):e0161386. doi: 10.1371/journal.pone.0161386. eCollection 2016

3. Demehri S, Belzberg A, Blakeley J, Fayad LM. Conventional and functional MR imaging of peripheral nerve sheath tumors: initial experience. AJNR Am J Neuroradiology 2014;35(8):1615-20.

\section{Figure Legends}

Figure 1: Note the fusiform bilobed mass (dashed arrows) with a medial necrotic and a lateral solid component, predominantly hypo-isointense (hypointense marked with white circle, isointense with white asterisk) to muscle in axial $(A)$, sagittal $(E)$ and coronal $(H) T 1-$ weighted (T1W) MRI sequences. Note abnormal gadolinium enhancement in axial $(B)$ and sagittal (F) postcontrast T1W sequences, and heterogeneous signal (black asterisk) in coronal (I) and sagittal (G) T2-weighted sequences. Diffusion Weighted Imaging (C) shows restricted diffusion (thin arrow) with low values (thick arrow) on Apparent Diffusion Coefficient imaging (D).

Figure 2: CT scan (A, B), ${ }^{18}$ Fluorodeoxyglucose positron emission tomography $\left({ }^{18} \mathrm{FDG}\right)$ PET (C, D) and Fusion-PET/CT ( ${ }^{18}$ FDG)-PET-CT (E and F) showing increased uptake and enlargement of the right sciatic nerve (indicated by arrow in $B, D$ and $F$ and targeted in $A$, $\mathrm{C}$ and $\mathrm{E})$. 


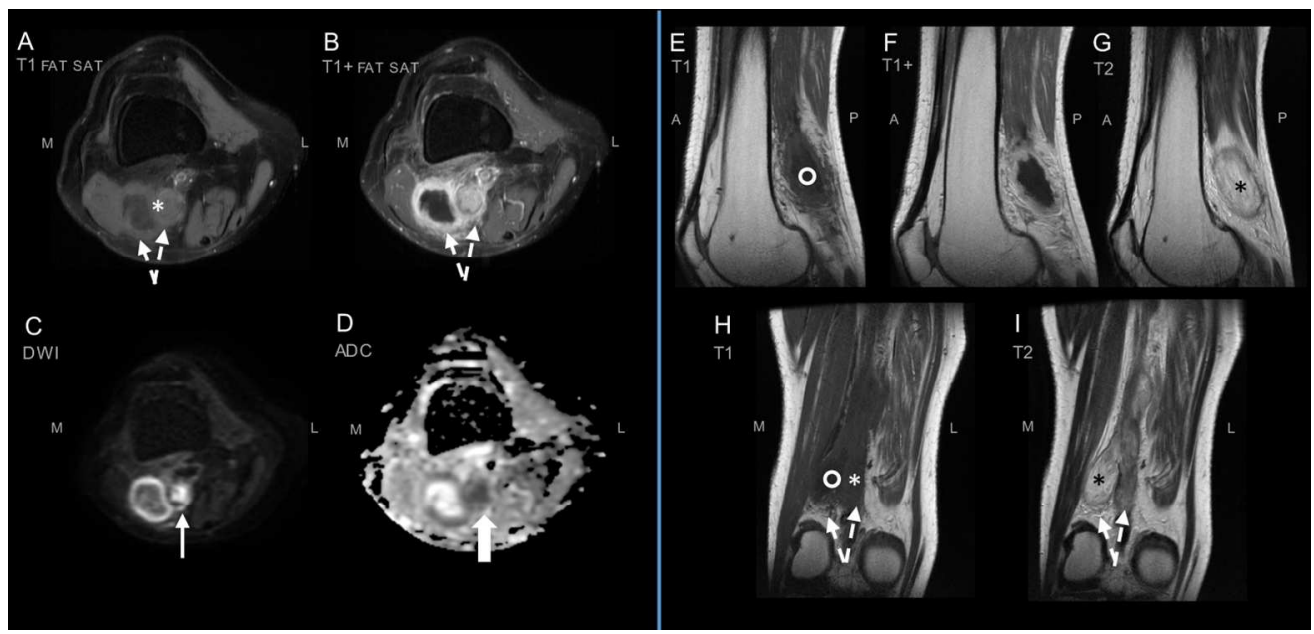

Figure 1: Note the fusiform bilobed mass (dashed arrows) with a medial necrotic and a lateral solid component, predominantly hypo-isointense (hypointense marked with white circle, isointense with white asterisk) to muscle in axial (A), sagittal (E) and coronal $(H)$ T1-weighted (T1W) MRI sequences. Note

abnormal gadolinium enhancement in axial (B) and sagittal (F) postcontrast T1W sequences, and heterogeneous signal (black asterisk) in coronal (I) and sagittal (G) T2-weighted sequences. Diffusion Weighted Imaging (C) shows restricted diffusion (thin arrow) with low values (thick arrow) on Apparent Diffusion Coefficient imaging (D).

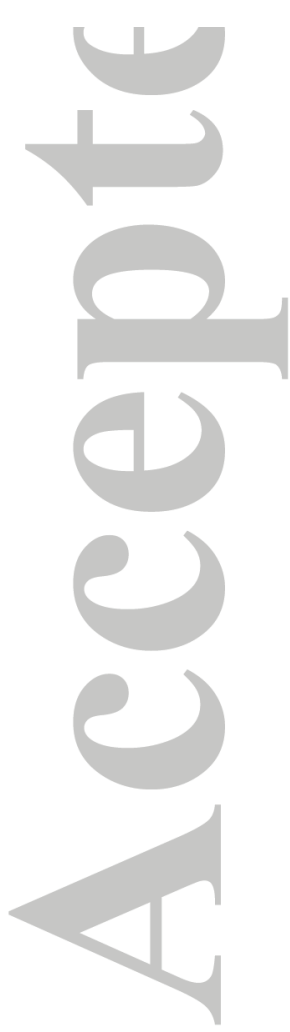

$130 \times 62 \mathrm{~mm}(300 \times 300 \mathrm{DPI})$

John Wiley \& Sons, Inc.

This article is protected by copyright. All rights reserved. 


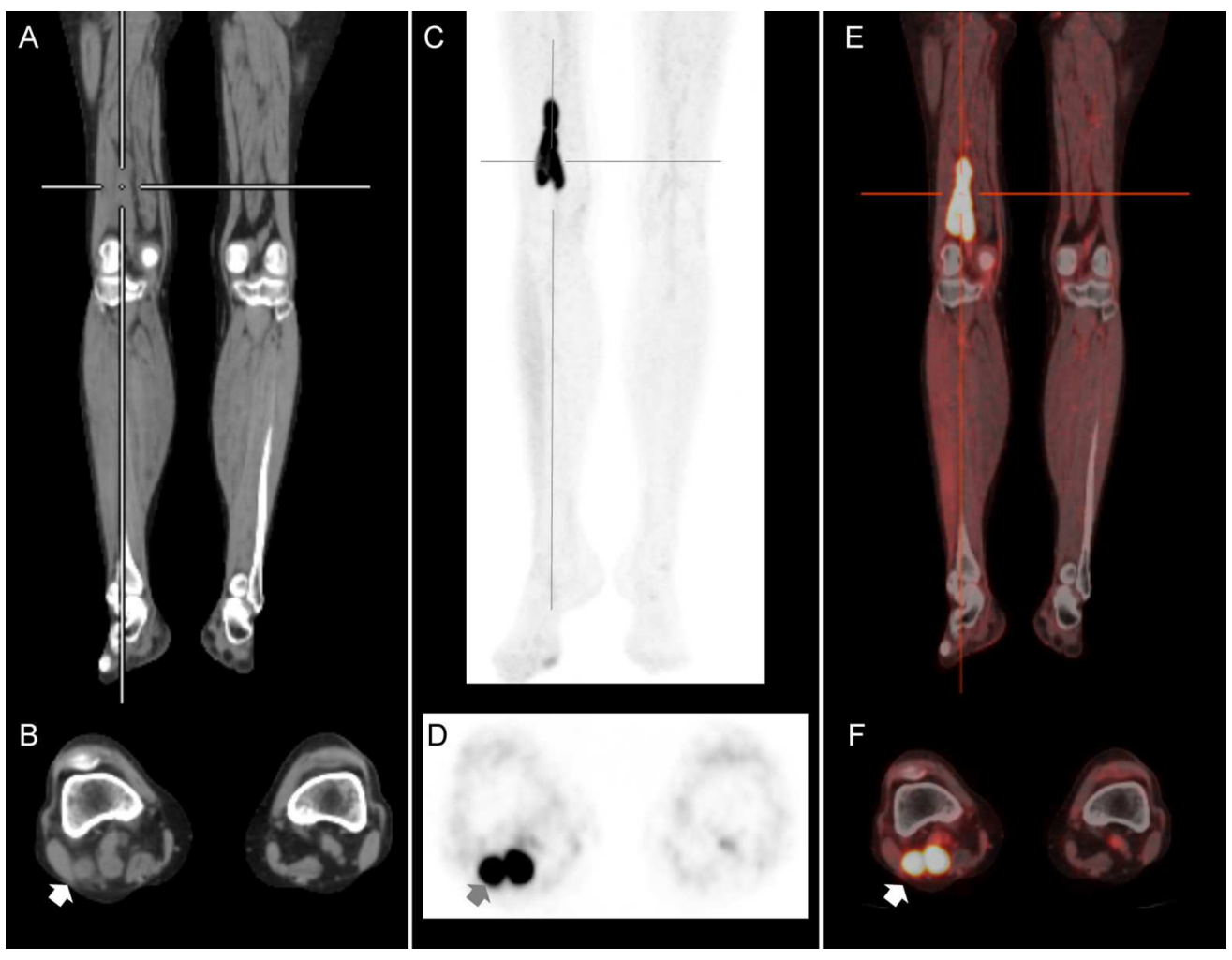

Figure 2: CT scan (A, B), 18Fluorodeoxyglucose positron emission tomography (18FDG)-PET (C, D) and Fusion-PET/CT (18FDG)-PET-CT (E and F) showing increased uptake and enlargement of the right sciatic nerve (arrowed in $B, D$ and $F$ and targeted in $A, C$ and $E$ ).

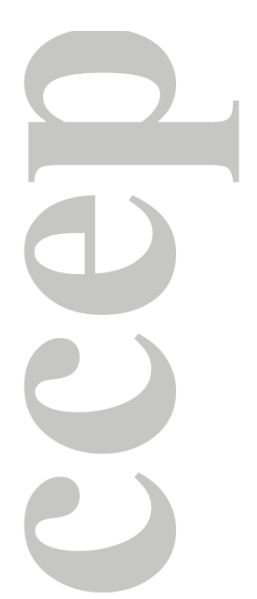

$129 \times 99 \mathrm{~mm}(300 \times 300$ DPI $)$

John Wiley \& Sons, Inc.

This article is protected by copyright. All rights reserved. 